\title{
Drying Characteristics of Preconditioned Rice in Fluidized Bed Dryer
}

\author{
Kumari Namrata* and S.K. Das \\ CAE Pusa, DR RPCAU Pusa Samastipur-848125, India \\ *Corresponding author
}

A B S T R A C T

\section{Keywords \\ Minimum fluidisation velocity, Puffed rice, Pre- conditioning, Expansion ratio, Amylose content \\ Article Info \\ Accepted: \\ 16 April 2018 \\ Available Online: \\ 10 May 2018}

\begin{abstract}
In this study, determination of minimum fluidisation velocity of rice, effects of temperature on drying rate and puffing quality of pre-conditioned rice were investigated. The experiments were conducted on two varieties of rice namely parboiled Polished rice namely medium and long. The drying behaviour in a fluidised bed was studied at three different temperatures of 50,60 and $70^{\circ} \mathrm{C}$ and the air velocity $3.6 \mathrm{~m} / \mathrm{s}$. Moisture reduced exponentially with time and dependent upon drying temperature. Minimum fluidisation velocity at 10 and $30 \%(\mathrm{wb})$ moisture content is 0.95 and $0.87 \mathrm{~m} / \mathrm{s}$ respectively. Puffing characteristics like Expansion ratio and \% puffing of low amylose content of rice was greater than more amylose content of rice. It was noticed that expansion of rice during puffing correlated strongly with amylose content.
\end{abstract}

\section{Introduction}

Rice, a leading food crops in the world, is expected to touch a record of 480.5 million tonnes (milled rice basis) in this year 2011 according to FAO. Though rice is mainly consumed as whole cooked grains, varieties of products are also prepared as breakfast foods, snacks and fermented products. It is reported that about $10 \%$ of the production of paddy is converted to three rice products, namely, puffed rice, popped rice and flaked rice (Chattopadhyay, 2004). Among the rice based breakfast cereals, puffed rice is largely demanded product for centuries. Its production mostly confined to unorganized rural sectors. Very recently, mechanized production system is being adopted to meet increasing domestic and export demands.
Puffing of rice is done by different methods, viz., conduction puffing on hot sand bed, convection heating in hot air or oil, explosion puffing using pressure differential (Chandrasekhar and Chattopadhyay, 1988). The puffing method traditionally followed in most part of India is sand-roasting, in which hydrothermally treated pre-conditioned rice (cured rice ready to puff) at 9 to $10 \%$ moisture content (wb) is roasted on hot sand at 250$260^{\circ} \mathrm{C}$ for 20-25 seconds (Jayasmita, 2008). Puffing of freshly prepared pre-conditioned rice using domestic microwave oven have been reported to give hygienic product with puffing as high as 95 - 100\% (Jayasmita, 2008). Further, puffing efficiency for rice grain depends on several factors including nature and concentration of salts diffused into the kernel (Chinnaswamy and Bhattacharya, 
1983b). Pre-conditioning of rice, a most critical factor for achieving the good quality expanded product, is basically a uniform and slow heating of high moisture (water soaked) parboiled grains in a large bowl coupled with turning or agitation system that facilitates proper structural changes like, surface modifications and hardness, and hence, highly expanded smooth-surface puffed rice (Chinnaswamy and Bhattacharya, 1983b; Chandrasekhar and Chattopadhyay, 1991).

Non-uniform heating of grain severely impairs the quality of product with less expansion ratio in addition to rough and blistered surface. This is tedious and laborious method of preconditioning but is cheap process.

A mechanized system, simulating the traditional process of pre-conditioning, has been reported recently (Minati, 2010). The system consisted of power driven regulated speed agitator assembly and electrical heating arrangement ensuring uniform preconditioning. This requisite condition of uniform heating and slow moisture transfer for pre-conditioning could also be carried out using a fluidised bed. Fluidized bed does not have any moving part, is simple in construction, and involves heating and drying of individual particle. No report in this aspect is available in the literature.

During pre-conditioning, the heating of saltdiffused high moisture rice kernels (around $35 \% \mathrm{db}$ ) is carried at around $70-90^{\circ} \mathrm{C}$ for about $70-90$ minutes so as to attain final moisture content of around $10 \% \mathrm{db}$ (Jayasmitha, 2008). This cured rice is now ready for puffing on hot sand bed/hot air for proper expansion and porous structure. Regulated flow rate and temperature of air can be employed to achieve the required fluidization, rate of heating, moisture reduction - all leading to proper preconditioning of rice.

\section{Materials and Methods}

\section{Materials}

The experiments were conducted on two varieties of rice namely parboiled Polished rice medium (Mugai) and long (Heera) with $\mathrm{L} / \mathrm{B}$ ratio of 2.3 and 3.13 respectively, specified for making puffed rice, were procured from the local market, Kharagpur, West Midnapur, West Bengal, India. These collected samples were used in various studies; these include preparation of preconditioned rice using fluidised bed dryer, control sample for puffing, determination of amylose content and determination of minimum fluidisation velocity.

\section{Experimental procedures}

Drying experiments were conducted in a fluidized bed dryer in a laboratory. The system mainly consists of a transparent conical flask, a blower, a thermostatically controlled electrical heater. Thermocouples. Thermostat. Butterfly valve, timer.

Preconditioned Rice was prepared by mixing $3.5 \%$ salt solution $(\mathrm{NaCl})$, soaked in distilled water and soaked rice was allowed to temper for $8-9$ hours for moisture and salt equilibration. The initial moisture content of the rice sample was determined by an oven drying method (AOAC, 1990) at $105^{\circ} \mathrm{C}$ for 24 hours using a hot air oven. The drying experiments of salt-diffused parboiled rice were carried out at temperatures of 50,60 and $70^{\circ} \mathrm{C}$ at an air velocity of $3.6 \mathrm{~m} / \mathrm{s}$. The drying was continued until the moisture content came around $10 \%(\mathrm{wb})$ as determined by the hardness of the kernel. Samples were collected from the dryer at 15 minutes intervals, each time they were collected in a moisture box and immediately used for moisture determination, hardness The hardness of the rice grain was around $45-50 \mathrm{~N}$ at $10-11 \%$ (wb) moisture 
content (Jaysmita,2008). Moisture content of the rice samples at different time intervals was determined according to the standard procedure (AOAC, 1990). About $2 \mathrm{gm}$ of rice was taken in a moisture box, weighed accurately (Sartorius BS323, Max capacity = $320 \mathrm{~g}$, accuracy $0.001 \mathrm{~g}$ ) and kept inside a hot air oven maintained at $105 \pm 1^{\circ} \mathrm{C}$ for $24 \mathrm{~h}$. Grain hardness was measured by a grain hardness tester (Kiya, Japan ; maximum load $20 \mathrm{~kg}$ with Least Count of $0.2 \mathrm{~kg}$ )

\section{Minimum fluidization velocity}

Measure weight of salt diffused rice sample was put inside the detachable transparent cylinder. The bed was vigorously shaken with high inlet air velocity and then was slowly reduced to zero velocity so that all the grains settled loosely and form a stable porosity bed.

This initial bed height was measured. Air flow was increased slowly and its effects on bed height and pressure drop were noted. This process continued until significant amount of grain assumed an escape velocity from the bed. The reverse process was also noted, viz., lowering the air flow rate gradually and pressure drop and bed height were noted till the bed returns to its original stable porosity bed. This experiment was replicated thrice using a fresh lot of the sample each time. The pressure drop and the air velocity were plotted in log-log paper to get the minimum fluidization velocity of the grain at different moisture contents. The whole experiment was carried out at ambient air temperature.

\section{Puffing}

All the puffing experiments of rice were carried out with a domestic microwave oven (SAMSUNG, model no: M197DL). During measurement of percentage of puffing, about $10 \mathrm{~g}$ pre-conditioned rice was accurately weighed in an electronic balance (Sartorius,
Model no. BS323 S, least count $=0.001 \mathrm{~g}$ ), poured into a packet and closed. It was subjected to puffing in the microwave oven for a definite period applying specified power level. The puffed and un-puffed grains were then separated and un-puffed grains were weighed. The corresponding numbers of initial grains $\left(\mathrm{N}_{\mathrm{PC}}\right)$ and number of un-puffed grains $\left(\mathrm{N}_{\mathrm{UP}}\right)$ were estimated. Then the percentage of puffing was calculated with the following equation (2.2).

Percent of Puffing $=\frac{\left(N_{p c}-N_{u p}\right)}{N_{p c}} \times 100$

The following experimental plan (Table 1) was followed for evaluating the puffing performance.

The expansion ratio (ER) of puffed rice is the ratio of the true volume of the puffed rice $\left(\mathrm{V}_{\mathrm{P}}\right)$ to the volume of pre-conditioned rice before puffing $\left(\mathrm{V}_{\mathrm{PC}}\right)$. Since the puffed rice is a porous material, measurement of its volume with gas displacement Pycno-meter will give erroneous result. Therefore, a separate methodology was followed to evaluate the true volume of puffed rice as reported elsewhere (Jayasmita, 2008).

\section{Results and Discussion}

\section{Effect of air temperature on moisture reduction during pre-conditioning}

The progressive reduction of moisture of saltdiffused rice is shown in Table 2. The Figure 1 is shown that the moisture reduction profiles at different temperature of drying. It was seen from this graph that there was no much difference between drying at 60 and $70^{\circ} \mathrm{C}$ up to 15 minutes of initial drying period; the drying for the later was found to be faster than the former. After 25 minutes of initial drying, the moisture reduction profiles for 50 and $60^{\circ} \mathrm{C}$ were quite close. 
Table.1 Experimental plan for evaluation of puffing characteristics of rice

\begin{tabular}{|l|c|l|}
\hline Independent variables & Levels & Values \\
\hline Microwave power, w & 3 & $600,850,1000$ \\
\hline Time of puffing, $s$ & 1 & 30 \\
\hline Dependent variables: Percentage of puffing, Expansion ratio \\
\hline
\end{tabular}

Table.2 Reduction of moisture content and grain hardness during pre-conditioning in a fluidized at different air temperatures

\begin{tabular}{|c|c|c|c|c|c|c|}
\hline \multirow{3}{*}{$\begin{array}{l}\text { Time } \\
\text { (min) }\end{array}$} & \multicolumn{6}{|c|}{ Temperature $\left({ }^{\circ} \mathbf{C}\right)$} \\
\hline & \multicolumn{2}{|c|}{50} & \multicolumn{2}{|c|}{60} & \multicolumn{2}{|c|}{70} \\
\hline & $\begin{array}{l}\text { M.C. } \\
\text { (\%wb) }\end{array}$ & $\begin{array}{l}\text { Hardness } \\
(\mathrm{N})\end{array}$ & $\begin{array}{l}\text { M.C. } \\
\text { (\%wb) }\end{array}$ & $\begin{array}{l}\text { Hardness } \\
(\mathrm{N})\end{array}$ & $\begin{array}{l}\text { M.C. } \\
\text { (\%wb) }\end{array}$ & $\begin{array}{l}\text { Hardness } \\
(\mathrm{N})\end{array}$ \\
\hline $\mathbf{0}$ & 25.6 & 11.45 & 25.5 & 11.48 & 25.76 & 11.27 \\
\hline 15 & 21.67 & 20.36 & 19.31 & 18.34 & 18.65 & 17.45 \\
\hline 30 & 17.91 & 26.61 & 17.56 & 26.98 & 14.87 & 33.12 \\
\hline 45 & 16.92 & 28.13 & 15.43 & 32.45 & 12.1 & 38.43 \\
\hline 60 & 13.65 & 34.91 & 12.76 & 37.34 & 10.3 & 46.87 \\
\hline 75 & 11.76 & 39.32 & 10.45 & 46.12 & - & - \\
\hline 90 & 10.3 & 47.08 & - & - & - & - \\
\hline
\end{tabular}

Table.3 Puffing characteristics of two variety of rice

\begin{tabular}{|c|c|c|c|c|}
\hline Rice & Power(W) & \% Puffing & E.R & Time(s) \\
\hline & 600 & 48.76 & 2.85 & 30 \\
\hline Heera & 850 & 73.78 & 4.06 & 30 \\
\hline & 1000 & 95.56 & 5.5 & 30 \\
\hline Mungai & 600 & 45.43 & 2.43 & 30 \\
\hline & 850 & 72.65 & 3.65 & 30 \\
\hline & 1000 & 92.34 & 4.96 & 30 \\
\hline
\end{tabular}


Fig.1 Change in moisture content in rice during preconditioning at different temperature and time

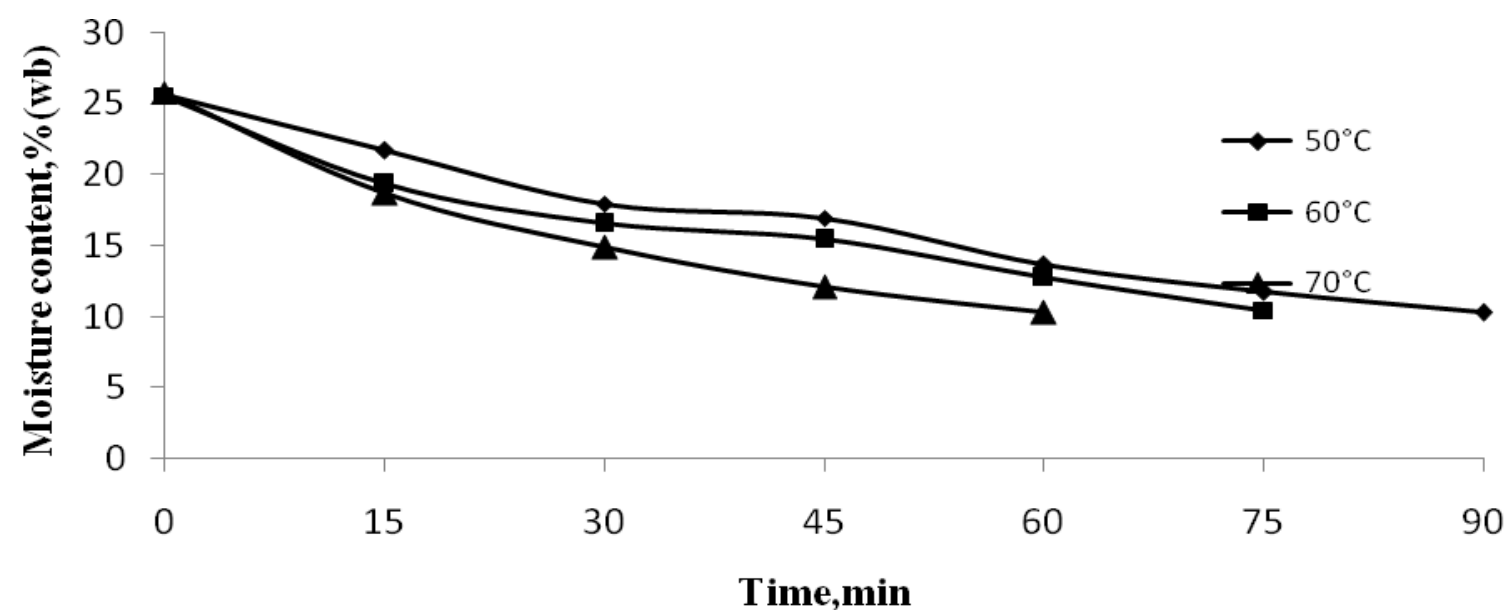

Fig.2 Variation in drying rate Vs average moisture content at different temperature

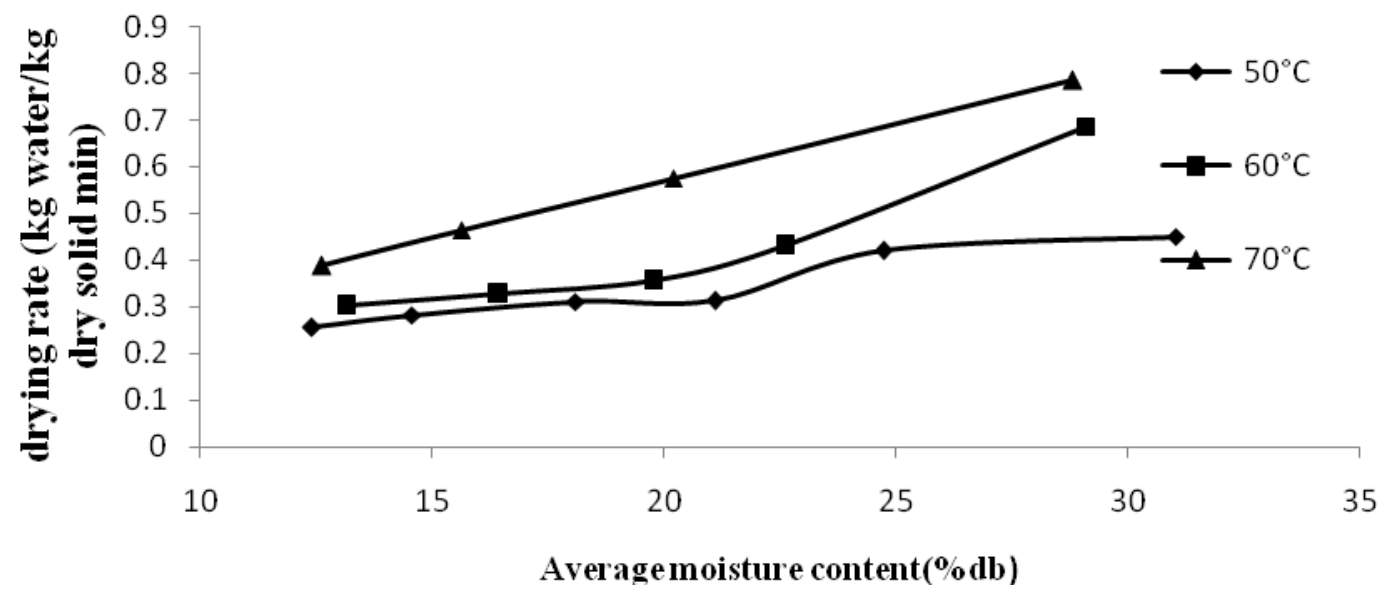

Fig.3 Variation in drying rate Vs time at different temperature

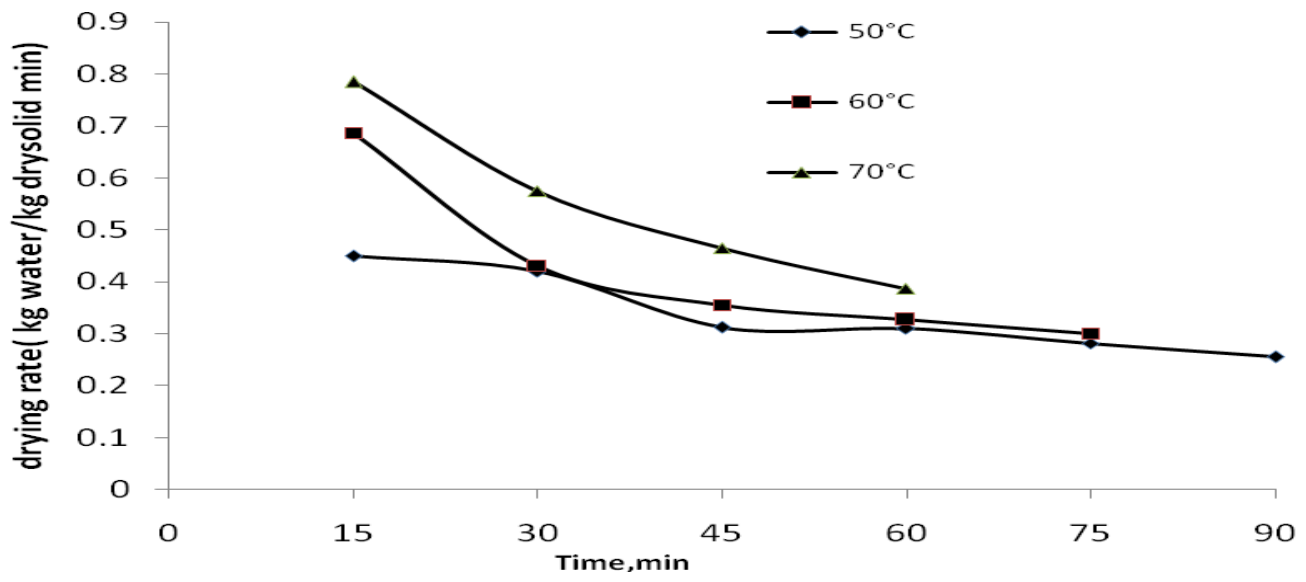


Fig.4 Comparison of expansion ratio of two varieties of pre-conditioned rice

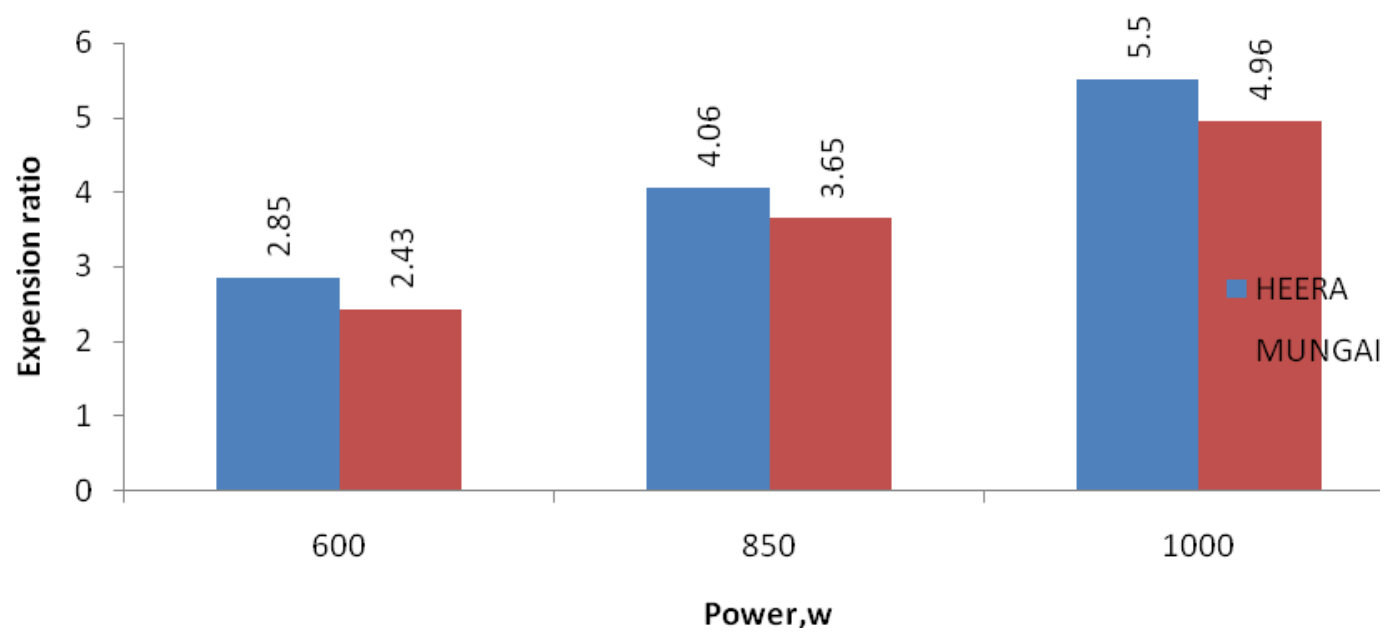

Fig.5 Comparison of percentage of puffing of two varieties of pre-conditioned rice

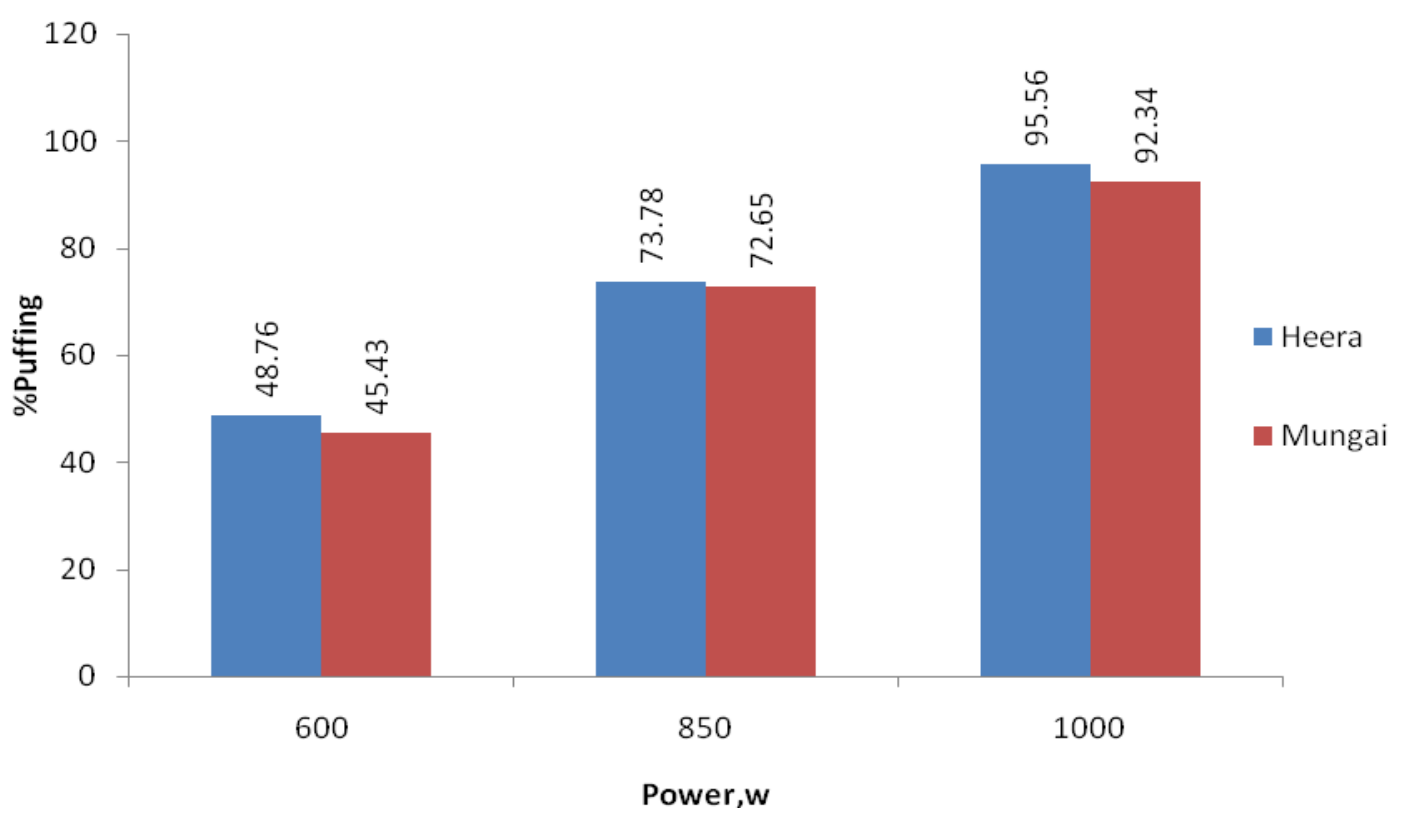

Variation in rates at different temperature of drying

The comparison of moisture reduction is shown by drawing plots of drying rate versus moisture content and drying rate versus time. The Figure 2 shows that drying rate is highest for $70^{\circ} \mathrm{C}$ temperature. After $20 \%$ of moisture content drying rate is almost same for 50 and $60^{\circ} \mathrm{C}$ Temperature. Figure 3 shows that drying rate is highest for $70^{\circ} \mathrm{C}$, temp up to $20 \mathrm{~min}$. It becomes almost constant for all temperatures after 20 minute.

Minimum fluidization velocity of rice kernel

The minimum fluidization velocity of rice were obtained from the experiment as $0.95 \mathrm{~m} / \mathrm{s}$ and $0.89 \mathrm{~m} / \mathrm{s}$ of rice kernel with an initial 
moisture content of $30 \%$ (wb) and final moisture content $10 \%$ (wb) respectively.

\section{Effect of puffing characteristics}

Amylose content of long (Heera) and medium variety (Mungai) of rice was found to be 21.43 and $23.12 \%$ is shown in Table 3 by using Spectro-photometer. It was observed from the Figure 4 and 5 that puffing characteristics like Expansion ratio and \% puffing of low amylose content of rice have more than medium variety of rice because amylose content of long variety of rice is $7.30 \%$ lower than that of Medium variety of rice. It is noticed that expansion of rice during puffing correlated strongly with amylose content.

\section{Acknowledgement}

I wish to express my sincere gratitude to my project supervisor Prof. S.K. Das, Professor, Department of Agricultural and Food Engineering, Indian Institute of Technology, Kharagpur for his constant supervision and guidance in carrying out this project work and thesis preparation.

I am thankful to Prof. P.B.S Bhadoria, Head, Department of Agricultural and Food Engineering, Indian Institute of Technology, Kharagpur for providing me the necessary facilities during the course of this dissertation program.

I sincerely wish to express my heartiest gratitude and deep sense of indebtedness to Prof. H. N. Mishra, Prof. T. K. Goswami,
Prof. P. P. Srivastava, Prof. G.C. Majumdar, Prof. M. Das, Prof. P. S. Rao, Prof A.K Datta, Prof. S.L. Shrivastava and other faculty members of the department for their encouragement and valuable suggestions during my project work.

Finally, I am unable to express my gratitude in words for the benevolence of almighty without which herculean task might have remained a distant dream to be accomplished

\section{References}

Chandrasekhar, P.R. (1989). Some studies on heated air fluidized bed-puffing characteristics of rice. Unpublished $\mathrm{Ph}$. D thesis, Indian Institute of Technology, Kharagpur, India.

Chandrasekhar, P.R. and Chattopadhyay, P.K. (1991). Rice puffing in relation to its varietal characteristics and processing conditions. Journal of Food Process Engineering 14 (4): 261-Chinnaswamy R. and Bhattacharya, K.R. (1983b) Studies on expanded rice, Optimum processing conditions. Journal of Food Science 48: 1604-1608.

Jayasmita (2008). Development of a rice conditioner for making puffed rice using domestic microwave oven. Unpublished M.Tech. Thesis, Indian Institute of Technology, Kharagpur, WB, India.

Mahapotra (2010). Mechanized preconditioning of rice and its microwave puffing. Unpublished Ph.D. Thesis, Indian Institute of Technology, Kharagpur, WB, India.

\section{How to cite this article:}

Kumari Namrata and Das, S.K. 2018. Drying Characteristics of Preconditioned Rice in Fluidized Bed Dryer. Int.J.Curr.Microbiol.App.Sci. 7(05): 1922-1928. doi: https://doi.org/10.20546/ijcmas.2018.705.225 